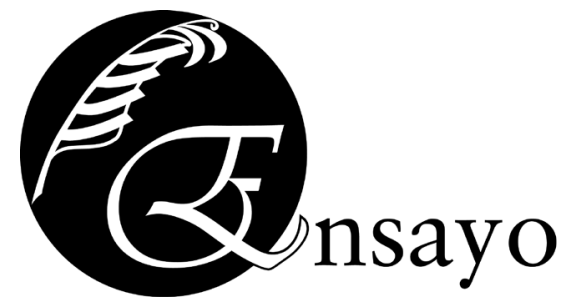

\title{
La tecnología: una herramienta de apoyo para PyMEs y emprendedores desde el entorno universitario
}

\author{
Ramón Ventura Roque Hernández*, Juan Manuel Salinas Escandón*, \\ Adán López Mendoza* y Juan Antonio Herrera Izaguirre*
}

Recepción: 4 de febrero de 2016

Aceptación: 2 de junio de 2016

*Universidad Autónoma de Tamaulipas, México. Correos electrónicos: rvHernandez@uat.edu. mx; jmSalinas@uat.edu.mx; aLopez@uat.edu.mx; jaHerrera@uat.edu.mx

Se agradecen los comentarios de los árbitros de la revista.

\section{Introducción}

La actividad empresarial y la tecnología están estrechamente vinculadas hoy en día. La tecnología apoya a casi todas las operaciones de negocios y resulta muy importante para el crecimiento de cualquier empresa. Sin embargo, en muchos casos, la tecnología no es explotada al máximo o

Resumen. Se analizan los medios para impulsar actuaciones innovadoras y revalorizar otras existentes con el objetivo de promover una cultura tecnológica que apoye al emprendimiento y a las empresas desde el entorno universitario. Se presentan diferentes tecnologías como herramientas valiosas para pequeñas y medianas empresas (PyMEs) y para emprendedores; asimismo, se discuten posibilidades de participación conjunta entre ellos y el sector académico y se exponen casos de éxito en la experiencia de los autores. Se destaca que la relación universidadempresa debería concebirse más como un enlace y menos como una brecha. En este sentido, las tecnologías son un elemento unificador, ya que son estudiadas e investigadas en la universidad y son requeridas en las empresas.

Palabras clave: tecnología, herramientas, emprendimiento, empresas, universidad.

\section{Technology: A Support Tool for SMEs (Small and Medium-Sized Enterprises) and Entrepreneurs from the University Environment}

Abstract. This paper analyzes the means to motivate innovative interventions and to reexamine some others with the aim of promoting a technological culture that supports entrepreneurship and enterprises from the university environment. Different technologies are presented as valuable tools for small and medium-sized enterprises (SMES) and entrepreneurs. In addition, joint participation possibilities between them and the academia are discussed, and success stories from the authors' experience are also introduced. It is highlighted that the relationship between university and enterprises should be conceived like a bridge instead of a gap. In this scenario, technologies are unifying blocks: universities focus their studies and research efforts on them while they are also being required by the enterprises.

Key words: Technology, Tools, Entrepreneurship, Enterprises, University.

no es valorada adecuadamente en el entorno empresarial, con lo que se pierden oportunidades de obtener los beneficios derivados de su uso.

En el entorno universitario, las Tecnologías de la Información y Comunicación (TIC) son un área transversal de estudio. Los estudiantes universitarios toman cursos acerca del uso de TIC como parte de su formación profesional.
Las tecnologías especializadas son abordadas en programas con una mayor acentuación en ciencias exactas como las licenciaturas o ingenierías en sistemas, ciencias de la computación o informática.

Por una parte, las empresas presentan una problemática de infrautilización tecnológica y, por otra, los estudiantes universitarios representan 
fuerzas tecnológicas activas en continua preparación que interactúan de diversas maneras con las empresas locales. Ahora bien, en la actualidad, no se pondera adecuadamente la relación universidad-empresa, la cual se concibe más bien como una brecha y no como un enlace del que pueden surgir beneficios importantes. Las empresas deben valorar seriamente su participación con el mundo académico; por su parte, las universidades deben promocionar con más intensidad su valor comercial y sus capacidades para apoyar a las Pequeñas y Medianas Empresas (PyMEs) a desarrollar ventajas competitivas (Dada y Fogg, 2016).

¿De qué manera será posible que todos estos actores puedan interactuar armónicamente para aprovechar nuevas oportunidades de desarrollo para el bien mutuo y el de la comunidad?, ¿cómo será posible propiciar nuevos escenarios de participación que involucren a estudiantes y empresas?, ¿cómo impulsar estas actuaciones innovadoras desde el entorno universitario y revalorizar esta participación dinámica? Para aproximarse a las respuestas a estas preguntas, primero hace falta dimensionar adecuadamente los elementos tecnológicos que serán el común denominador entre las empresas y la universidad.

Por lo anterior, este artículo tiene los siguientes objetivos: a) describir componentes tecnológicos importantes que las empresas y emprendedores deberían conocer y aprovechar y b) destacar medios para impulsar actuaciones innovadoras y revalorizar otras existentes para promover una cultura tecnológica que apoye al emprendimiento y a las empresas desde la universidad.

El contenido se encuentra estructurado de la siguiente manera: primero se abordan algunos aspectos tecnológicos para las Pymes. Posteriormente, se ex- ponen tecnologías importantes para los emprendedores. En el siguiente apartado se discuten algunos escenarios de apoyo a las pymes con tecnología desde el entorno universitario. Después se exponen acciones para contribuir con tecnología al emprendimiento desde la universidad; luego, se presentan tres casos de éxito en la vinculación universidad-empresas y finalmente se presentan las conclusiones.

\section{Tecnología en las PyMES}

Para el componente tecnológico de una estrategia de negocios, las empresas deben considerar al hardware (equipos y dispositivos), al software (programas y datos) y al recurso humano especializado. Una empresa debe invertir en estos elementos de manera estratégica y debe tomar decisiones importantes acerca de ellos.

En cuanto al hardware pueden requerirse por ejemplo: servidores, equipos de telecomunicación, equipos de cómputo personales de escritorio o móviles y dispositivos periféricos. En cuanto al software, por ejemplo, pueden ser necesarios sistemas operativos, aplicaciones ofimáticas y de seguridad, programas de gestión empresarial, así como aplicaciones especializadas en áreas de interés para la empresa.

Ante la disyuntiva de comprar equipo de cómputo, o bien rentarlo, se debe considerar que la compra resulta más económica que la renta durante un largo periodo, además se tiene la posibilidad de modificar o actualizar los equipos cada que sea necesario; estos gastos son deducibles fiscalmente en la mayoría de los casos. Por otra parte, el costo inicial de la adquisición del nuevo equipo tiende a ser alto y la empresa tiene la responsabilidad completa del equipo durante su tiempo de vida y hasta su obsolescencia. La renta de equipo podría ser atractiva, ya que no hay tanta inversión inicial como en el caso de la compra y el costo generalmente incluye pólizas de seguro, servicios de mantenimiento y actualización. Sin embargo, la empresa nunca es dueña de los equipos rentados y pueden existir costos de penalización si el contrato se da por terminado antes de la fecha acordada.

En relación con el software, existe la posibilidad de utilizar programas comerciales propietarios, por los cuales la empresa debe pagar o se puede utilizar software libre. Hoy en día muchos programas útiles están disponibles gratuitamente de manera legal en internet para su descarga y uso.

Los programas especializados para llevar las transacciones del negocio pueden ser adquiridos comercialmente o desarrollados de manera exclusiva para las necesidades de la empresa. Si se compran programas disponibles en el mercado, éstos suelen ser muy confiables, funcionales, económicos y con respaldo de la compañía que los comercializa; sin embargo, las funciones que ofrecen podrían no adaptarse totalmente a las necesidades de la empresa que los adquiere y no se tiene la certeza de que su distribuidor siga en el mercado para siempre. Por otra parte, de acuerdo con Kendall y Kendall (2013), el software que se desarrolla exclusivamente para una empresa da respuesta especializada a sus necesidades y le puede brindar ventajas sobre sus competidores por su grado de innovación; sin embargo, su proceso de creación es costoso, ya que se requiere personal para desarrollar el software y hacerle ajustes continuamente.

Una opción tecnológica que debería ser considerada por todas las empresas es la computación en la nube, que consiste en el uso de aplicaciones de negocios que son accedidas a través de internet 
desde diferentes plataformas. La infraestructura que se utiliza puede estar geográficamente distante de la ubicación de la empresa y ser rentada a través de un proveedor de servicios que se encarga de las labores técnicas relacionadas con esta arquitectura. Como las capacidades contratadas con el proveedor pueden aumentar o disminuir con poco esfuerzo, estas arquitecturas representan una ventaja desde la óptica empresarial.

Según el trabajo de Urueña et al. (2012) y Deloitte (2010), las soluciones basadas en la nube pueden hacer que una empresa ahorre hasta $50 \%$ de sus gastos en tecnología debido a que proporcionan funcionalidad nueva sin la necesidad de invertir en la compra de nueva infraestructura. Por otra parte, al delegar al proveedor de servicios de la nube las responsabilidades de operación, configuración y mantenimiento se requiere menos personal informático que labore directamente en la empresa.

En la literatura se ha reconocido que las tecnologías son un factor crítico para la administración de las empresas pequeñas, ya que proporcionan ventajas importantes (Alonso-Almeida y Llach, 2013) y que es necesario conocerlas para aprovecharlas mejor. Por ejemplo, en el trabajo de Wang y Wang (2015) se destacan algunas áreas de conocimiento tecnológico orientadas al usuario final que deberían ser enseñadas a las pequeñas empresas en un curso diseñado especialmente para ellas: $a$ ) computación y arquitecturas para pequeños negocios, $b$ ) sistemas para el soporte de decisiones, $c$ ) inteligencia de negocios, $d$ ) medios sociales para pequeños negocios, e) computación en la nube y $f$ ) software de código abierto. Estos conocimientos contribuirían a la explotación estratégica de la tecnología y derivarían en beneficios para las pequeñas y medianas empresas.

\section{La tecnología para el emprendedor}

\section{1. Emprendedor no especialista en tecnologías}

Los emprendedores requieren de un conocimiento básico de tecnologías y de una visión de alto nivel de las posibilidades tecnológicas más especializadas que están a su servicio. Por ejemplo, resulta imprescindible el conocimiento de herramientas ofimáticas como procesador de textos, hoja de cálculo y presentaciones electrónicas, así como el manejo de internet, el correo electrónico, las videollamadas y las transacciones en línea. También es muy importante saber utilizar por lo menos un sistema operativo y conocer aspectos básicos de seguridad informática como manejo de contraseñas, amenazas informáticas y respaldos.

Un emprendedor también requiere conocer medios para proporcionar una identidad digital a su empresa u organización. Esto se logra mediante redes sociales, administradores de contenido o portales web. Este conocimiento le permitirá diseñar e implementar estrategias de negocio y de mercadotecnia en línea, lo cual será la base para el desarrollo de su negocio. Otras herramientas que pueden resultar útiles son software para administración, planeación, manejo de riesgos y control de transacciones.

La capacitación tecnológica que recibe un emprendedor debería ir más allá del simple uso de tecnologías aisladas. Debería estar orientada a la satisfacción de necesidades específicas que actúen sinérgicamente para el cumplimiento de sus objetivos empresariales.

Si el emprendedor por sí mismo no tiene una idea clara acerca de las tecnologías que ayudarían a su idea de negocios, es necesario que busque asesoría con especialistas del área o con organismos de apoyo empresarial, quienes le pueden brindar información sobre las mejores formas de aprovechar los recursos tecnológicos para el éxito de su proyecto.

\section{2. Emprendedor especializado en tecnologías}

Para el emprendedor relacionado con las áreas técnicas más especializadas, las tecnologías que requiere dependerán de la orientación de su negocio. Una posibilidad de emprendimiento es en el área de desarrollo de software, que consiste en la elaboración de aplicaciones informáticas. En este escenario, un desarrollador debe conocer entornos de desarrollo, lenguajes de programación, herramientas para generar reportes y manejadores de bases de datos. Otra alternativa es en el área de mantenimiento de equipo y redes, en donde el emprendedor ofrece la instalación, mantenimiento y venta de equipos de cómputo, periféricos y accesorios. En este caso, el emprendedor debería conocer el uso de sistemas operativos, utilerías, software de configuración, diagnóstico de fallas y detección de amenazas.

Si el emprendedor desea ofrecer servicios relacionados con el diseño gráfico, sus habilidades deberían incluir el manejo de herramientas de manipulación fotográfica y de diseño electrónico de publicaciones. Por otra parte, en el campo de la creación de sitios web, un emprendedor debería contar con herramientas de diseño gráfico y de creación de contenidos para internet, así como también herramientas para administración de servidores web, transferencia de archivos y manejadores de bases de datos para internet. Si la asesoría y capacitación es su área de emprendimiento, el profesional necesitaría software para presentaciones electrónicas, manejadores de contenido, plataformas 
de aprendizaje, así como captura y edición de imágenes y video. Además de los ejemplos presentados, existen muchos otros perfiles profesionales que los alumnos pueden adoptar una vez que egresan de su carrera; incluso, en el futuro, las opciones de emprendimiento se incrementarán aún más con las nuevas especializaciones que irán surgiendo como consecuencia de las tendencias y avances tecnológicos.

\section{Apoyando a las PyMEs con tecnología desde el entorno universitario}

Desde el interior de la universidad se puede apoyar a las pymes en el área tecnológica. Este apoyo resulta valioso para las empresas, para los estudiantes y para la universidad. En este apartado se exploran algunas posibilidades de intervención. La lista de escenarios que se presenta no pretende ser exhaustiva sino destacar áreas potenciales de oportunidad.

\section{1. Prácticas o residencias profesionales}

Como un requisito para titularse, a los alumnos de las carreras universitarias se les solicita completar una estancia de varios meses de práctica profesional en alguna empresa de la localidad. Durante este tiempo, los alumnos trabajan en su área de especialización en un entorno real al mismo tiempo que ganan experiencia y establecen nuevas relaciones y contactos de negocios. Este periodo generalmente culmina con la contratación definitiva de los alumnos, para quienes en muchas ocasiones sería su primer empleo. Las empresas se benefician con la incorporación de personal universitario capacitado mientras que los estudiantes obtienen experiencia y remuneración económica en una actividad formal relacionada con su área de estudio.
Las prácticas profesionales permiten que la universidad colabore con las PyMES en el área tecnológica proporcionándoles personal capacitado que pueda apoyarles para aprovechar al máximo sus recursos. En el caso de carreras de sistemas o informática los alumnos se pueden incorporar directamente al departamento de tecnologías en tareas de desarrollo de software, mantenimiento, telecomunicaciones o soporte. En otras carreras con menos acentuación técnica, los alumnos pueden ingresar a departamentos de su área de especialidad colaborando con el manejo de herramientas tecnológicas propias de su carrera, por ejemplo, sistemas administrativos y contables, software de consulta y gestión aduanera.

\section{2. Abordar en las materias contenidos relevantes para las Pумеs} Otra manera de colaborar con las PyMEs desde el entorno universitario es que se aborde en las aulas los contenidos más relevantes para las empresas. Para lograr esto, se debe reconocer que la universidad es parte de un entorno socioeconómico y que debe responder a las necesidades de otros actores que forman parte de ese entorno. También es necesario conocer a las pyMEs en su filosofía, operación actual y requerimientos tecnológicos. El establecimiento de vínculos de comunicación y participación entre la universidad y las empresas resulta imprescindible en la determinación de estas necesidades. La universidad debe incorporar a sus programas de estudio temas afines a la realidad tecnológica y de negocios que viven las PyMEs y debe encaminar sus esfuerzos para que los estudiantes realicen tareas y proyectos alineados con esa realidad.

\section{3. Asesorías formales}

Las asesorías formales son mecanismos institucionales o legales y pueden estar compuestas por convenios, investigaciones, patentes, licencias o pago de regalías. Estas actividades deberían ser parte integral de la vinculación universidad-empresa; sin embargo, en ciertas ocasiones las asesorías formales no fluyen de manera adecuada hacia las PyMES debido a factores que inhiben la transferencia formal de conocimiento, siendo uno de ellos la premisa acerca de cómo las autoridades universitarias definen, administran y promueven estos procesos de asesoría (Rhoades y Slaughter, 1991).

En las asesorías formales se transfiere conocimiento hacía las distintas áreas funcionales que el pequeño empresario requiere: contabilidad, finanzas, mercadotecnia, aspectos fiscales y regulatorios, así como también tecnologías de información, las cuales podrían proporcionar ventajas estratégicas a la empresa por medio de la innovación de procesos o la mercadotecnia apoyada en las redes sociales.

\section{4. Asesorías informales}

Por asesoría informal se entiende el flujo de conocimiento de los procesos funcionales de las empresas, así como de tecnologías de información a través de un proceso de comunicación informal. Si bien existe una asistencia técnica, consultoría e investigación colaborativa, ésta surge a partir de una iniciativa personal de los académicos o de los microempresarios que la proponen o la solicitan de manera particular. En este tipo de transferencia tecnológica, los derechos de propiedad intelectual poseen un rol secundario y las obligaciones son más bien de carácter normativo, económico y basado en la confianza o amistad entre académicos y empresarios. Algunos académicos se apoyan para este tipo de asesoría en los alumnos de semestres avanzados en áreas como contabilidad, recursos humanos o tecnologías, por ejemplo. 


\section{5. Participación en proyectos de investigación y desarrollo}

Las empresas pueden realizar proyectos de investigación o desarrollo tecnológico en colaboración con las universidades. En este tipo de proyectos se cuenta con un fondo económico aportado total o parcialmente por una entidad pública o privada. Para realizar el proyecto se deben definir objetivos, realizar un protocolo de investigación y hacer una solicitud formal. Un comité evaluador se encarga de seleccionar los proyectos más factibles de concretarse para otorgarles los fondos con el compromiso de que los participantes entreguen los productos pactados.

Durante el desarrollo del proyecto se realizan actividades encaminadas al logro de los objetivos planteados en el protocolo. Algunos de los productos académicos que se derivan de estas actividades son libros, capítulos de libro, artículos, ponencias y tesis. Las empresas pueden obtener productos como software, maquinaria, procesos o conocimiento nuevo generado a través de la práctica. Los proyectos de investigación y desarrollo brindan oportunidades de participación conjunta para las empresas y las universidades que no deberían ser desaprovechadas porque expanden el conocimiento y lo aplican en actividades prácticas que son benéficas para todos.

\section{6. Eventos}

El entorno universitario es el lugar idóneo para organizar conferencias, congresos, talleres, cursos, exposiciones y ferias con temáticas tecnológicas de actualidad que resultan de interés para las PyMES y para los académicos. Estos eventos crean y fortalecen el vínculo entre la universidad y las empresas al mismo tiempo que proporcionan foros para divulgar información importante. En estos eventos, por ejemplo, se pueden presentar nuevos productos, discutir tendencias actuales, exponer áreas problemáticas o de riesgo, brindar alternativas de servicios o difundir resultados y proyectos en proceso. Cualquiera que sea la modalidad elegida, siempre resulta útil mantener actualizados los datos de las empresas participantes para asegurar la continuidad en la comunicación con ellas.

\section{Apoyar al emprendimiento con tecnología desde el entorno universitario}

Un emprendedor es aquel que asume el riesgo de iniciar un negocio o empresa propia cuyo objetivo es la venta de un producto o servicio, así como obtener una utilidad en la operación. En los negocios, el riesgo está directamente relacionado con la incertidumbre que emerge de la inversión en un producto o servicio sin el $100 \%$ de seguridad de venta o utilidad (Messerschmitt y Stuck, 2008). A continuación se examinan algunas maneras de apoyar a los emprendedores a asumir riesgos y tomar las decisiones más informadas incorporando la tecnología en su visión de negocios.

\section{1. Materias o cursos de emprendimiento}

La educación sobre emprendimiento proporciona valiosas habilidades a la juventud y le da la posibilidad de crear sus propios empleos (Premand et al., 2016). En la literatura se ha identificado que el emprendimiento puede ser enseñado y que esta educación puede ser considerada uno de los elementos más importantes para impulsar actitudes, intenciones y competencias emprendedoras (Karimi et al., 2016). Los cursos universitarios son una excelente oportunidad para impulsar directa o indirectamente el uso de las tecnologías en el proceso de creación de nuevas empresas.

\section{2. Proyectos emprendedores en las materias universitarias}

Martínez-Caro y Cegarra-Navarro (2012) proponen el desarrollo de proyectos de emprendimiento en las materias con tres objetivos: el primero por ser un medio de un aprendizaje activo, el segundo para adquirir las competencias transversales necesarias y el último para establecer una tendencia de fomento al espíritu emprendedor en los jóvenes universitarios.

Conforme a Ruiz Jiménez et al. (2012), en las materias de administración y contabilidad de los semestres avanzados es recomendable implementar prácticas de emprendimiento. En ellas los alumnos podrían realizar algunas de las distintas etapas de un plan de negocios y familiarizarse con las actividades de creatividad empresarial que se deberían tomar en cuenta al momento de iniciar un negocio. Lo anterior tendría como meta la motivación y la familiarización de los estudiantes con actividades de emprendimiento dentro del contexto universitario, en donde los estudiantes ubiquen oportunidades para iniciar un negocio y desarrollen competencias emprendedoras que aporten soluciones de autoempleo y de esta forma generar un impacto económico en el país. Sin importar el perfil de las materias en las que se promueven estos proyectos, bien puede impulsarse el uso de la tecnología como un elemento principal o de soporte en la propuesta realizada por los estudiantes.

\section{3. Conferencias y eventos de emprendimiento}

Las conferencias y eventos de emprendimiento en donde estén presentes estudiantes, empresarios e inversores ángel (Ding et al., 2014) pueden ser útiles para todos los actores involucrados en esta práctica, tanto desde el punto de vista de conocimiento y experiencias a adquirir, asî 
como para la práctica de socialización que pudiera resultar en contactos clave para apoyar financieramente los proyectos de los estudiantes o para que el futuro emprendedor reciba puntos de vista de otras personas que ya están inmersas en la actividad empresarial.

Ya que las tecnologías de la información están identificadas como elementos benéficos para las empresas y el emprendimiento, resulta muy importante incluir temas afines en las conferencias y eventos que se organicen con el objetivo de contribuir a la creación de la buena cultura tecnológica.

\section{Casos de éxito}

A continuación se describen tres casos de éxito que los autores han experimentado en el marco de este artículo.

\section{1. Servicios informáticos}

Estudiantes voluntarios de licenciatura en informática han ofrecido servicios gratuitos de instalación de software, así como de configuración y mantenimiento de equipos personales a la comunidad universitaria como parte de eventos académicos semestrales que se realizan en su carrera, en donde también hay conferencias y talleres relacionados con la tecnología. La idea inicial surgió entre ellos como una inquietud de servicio y se apoyó como un ejercicio para impulsar el emprendimiento en el área tecnológica. Esta experiencia resultó muy positiva, ya que los alumnos trabajaron en este proyecto como si se tratara de su propio negocio. Ellos definieron horarios de servicio, difundieron información del evento, organizaron equipos de trabajo, establecieron turnos y estrategias para administrar las órdenes de servicio, interactuaron con usuarios con problemáticas reales y propusieron e implementaron soluciones prácticas. También se auxiliaron de herramientas de software que ellos mismos configuraron para llevar el control de los servicios y reparaciones realizadas.

Como resultado, los alumnos dijeron haberse enriquecido con ideas, experiencias y formas de trabajo. También expresaron que se sentían motivados para emprender un negocio propio relacionado con la venta, instalación y el mantenimiento de equipo de cómputo puesto que habían detectado necesidades importantes entre los solicitantes de sus servicios y percibían que sería una buena oportunidad para ganar dinero y generar empleos.

Para el éxito de este evento fue determinante el apoyo que brindaron tanto la dirección de la facultad como la Coordinación de la Licenciatura en Informática. Ambas entidades se encargaron de gestionar espacios, horarios, herramientas y recursos necesarios para que los alumnos llevaran a cabo esta actividad. Otro aspecto relevante fue que los alumnos emprendedores estuvieron comprometidos todo el tiempo con su labor. En ningún momento desatendieron a quienes solicitaban sus servicios. También fue muy importante la difusión que tuvo el evento en la universidad por medio de carteles, mantas y redes sociales; asimismo, se visitó cada una de las aulas en el campus para dar a conocer los detalles de los servicios proporcionados, los horarios y las ubicaciones. Por otra parte, es destacable la confianza que los universitarios tuvieron en estos servicios de cómputo, ya que fueron muchas las computadoras que fueron diagnosticadas, configuradas o reparadas.

\section{2. Exposición de proyectos de software}

Los alumnos también han participado en exposiciones semestrales de pro- yectos de desarrollo de software. En estos eventos, los alumnos presentan los programas de cómputo que ellos mismos elaboran atendiendo algunas necesidades de negocios reales o proponiendo soluciones o ideas innovadoras a través del software. Algunos de estos estudiantes han recibido una remuneración económica por parte de los negocios para los que se desarrolló el software; otros, además de vender su programa, pudieron ofrecer servicios de mantenimiento, capacitación y actualización por varios meses posteriores a la entrega del software final.

Durante la exposición, todos los proyectos se presentan al mismo tiempo en diferentes computadoras. Los visitantes realizan un recorrido por los trabajos de los alumnos, observan y los retroalimentan con sus comentarios. A estas exposiciones han asistido, además de la comunidad universitaria, destacados profesionistas emprendedores en el área del software, quienes han establecido contacto con alumnos y maestros. Como producto de esa relación se han concretado algunas contrataciones y se han establecido vínculos con el sector empresarial local. Las contrataciones han permitido que los alumnos de los últimos semestres se desempeñen en el área de desarrollo de software y vayan ascendiendo con pasos firmes en su trayectoria profesional. En otras ocasiones, lo que inicia entre los estudiantes y los visitantes es una relación de negocios que satisface la necesidad de servicios informáticos principalmente en el área de programación de aplicaciones.

Algunos factores que han resultado muy importantes para el éxito de estas exposiciones han sido el apoyo institucional con el que se ha contado, la seriedad con la que los visitantes externos han tomado estos eventos, así como la iniciativa y la responsabilidad de los estudiantes que exhiben su trabajo. 


\section{3. Expo Proveedores}

Otro evento que ha resultado muy positivo para fomentar tanto el emprendimiento como la relación entre universidad y empresas es la Expo Proveedores, una exhibición informática que se ha realizado dentro del campus universitario. A este evento han asistido proveedores de productos y servicios de cómputo de la localidad con el objetivo de dar a conocer su actividad empresarial a los estudiantes. Entre los participantes hay vendedores de equipo de cómputo, accesorios y software, así como proveedores de servicios de reparación, actualización, mantenimiento y capacitación. Todos ellos son en su mayoría microempresarios o emprendedores entusiastas que han logrado posicionar exitosamente su negocio en la localidad. Por una parte, los expositores promueven su negocio entre los estudiantes, quienes podrían convertirse en clientes potenciales $\mathrm{o}$, incluso, si se encuentran ya laborando profesionalmente, podrían recomendar estos proveedores en sus lugares de trabajo. Por otra parte, los estudiantes conocen las posibilidades empresariales en el área tecnológica y aprenden de ellas. Durante este encuentro con las empresas, los alumnos realizan preguntas sobre los productos y servicios, pero también sobre las experiencias de negocio de los expositores. El diálogo resulta muy provechoso: los expositores conviven informalmente con los estudiantes $\mathrm{y}$ les comparten algunas de sus vivencias con sus proyectos emprendedores o microempresas. Existe mucha disposición de los expositores para atender a los estudiantes. Ha sucedido que algunos de ellos han estudiado en la misma universidad o carrera en la que hoy se encuentran los alumnos asistentes. En estos encuentros siempre hay intercambio de folletos y tarjetas de presentación, de tal manera que se promueve la vinculación entre la universidad y las empresas. También se sabe que algunos alumnos se han incorporado al trabajo en las empresas de los expositores como consecuencia de este acercamiento.

Sin duda uno de los factores más importantes que han incidido para el éxito de este evento ha sido la respuesta positiva que se ha obtenido por parte de los expositores. Ellos han acudido puntualmente al evento, han preparado sus lugares de exhibición y han atendido de manera diligente a los visitantes. El apoyo de la universidad es otro factor de éxito; a través de la dirección, de la coordinación de informática, de los maestros de la carrera y de los alumnos asistentes, se han logrado reunir todos los elementos necesarios para realizar esta exhibición.

\section{Reflexión}

En los tres casos que se han presentado puede observarse que el apoyo institucional, la colaboración estudiantil y la disposición empresarial aunados al trabajo docente comprometido han incidido para el éxito de los eventos. Si alguno de estos elementos fallara, sería difícil el desarrollo de cualquier iniciativa. Por lo tanto, uno de los principales retos en la organización de estos foros es la conjunción de todos los actores en un escenario colaborativo. Además, algunos de los problemas que pueden surgir en este contexto son la falta de compromiso, la apatía, la inmadurez y la incapacidad para asumir roles de algunos colaboradores. Estos inconvenientes pueden ser superados fácilmente con el trabajo en equipo, la iniciativa, las buenas actitudes, la buena voluntad y el apoyo de otras personas.

Por otra parte, es difícil cuantificar los beneficios obtenidos con los tres casos de éxito que se presentaron, pues no se tienen registros oficiales de logros ni estudios de seguimiento. Sin embargo, sí se cuenta con referentes informales que provienen de empresarios, maestros y alumnos. Resulta gratificante ver que en cada nueva generación de estudiantes es más frecuente encontrar actitudes emprendedoras y vinculaciones con el sector empresarial que enriquecen el perfil profesional de los involucrados. También resulta motivante observar que los alumnos cada vez se involucran más en el uso de las nuevas tecnologías y que las pequeñas y medianas empresas abren sus puertas a los estudiantes cada vez con mayor confianza.

\section{Conclusiones}

En este trabajo se analizaron las tecnologías como un eslabón entre la universidad, el emprendimiento y las pymes, se discutieron diferentes posibilidades de participación entre la comunidad universitaria y el mundo empresarial y también se destacó la importancia de la existencia de una relación dinámica entre ellos.

El mundo académico debe sensibilizarse acerca de su responsabilidad para promover una cultura tecnológica que apoye a las empresas, al emprendimiento y al desarrollo regional. Tanto el emprendimiento como la tecnología tienen que ser filosofías que acompañen al estudiante durante toda su formación y no únicamente en algunos semestres de su carrera profesional. También es labor de la universidad orientar los conocimientos, habilidades y valores que se promueven en el aula para que sean compatibles con la realidad actual de las empresas y del país. Entre los estudiantes universitarios se deberían fomentar actividades que fortalezcan la 
iniciativa, la persistencia, la confianza y el apoyo hacia actitudes emprendedoras, así como también el manejo de tecnologías, la investigación, el análisis de riesgos, la orientación a la calidad y la administración de recursos.

Estas acciones deberían realizarse en un marco activo de participación empresa-universidad que incluya nuevas actitudes orientadas a concretar prácticas colaborativas. Las tecnologías son un elemento unificador en esta nueva valorización del vínculo empresa-universidad, ya que al ser estudiadas e investigadas en la universidad y requeridas en la empresa representan un eslabón fundamental para la realización de actividades productivas relevantes y pertinentes.

\section{Análisis prospectivo}

En los próximos años la tecnología seguirá siendo una herramienta determinante para el desarrollo académico y empresarial. Los esfuerzos para vincular la universidad con las empresas y los emprendedores deben definirse desde ahora considerando a la tecnología como un componente que brinda variadas posibilidades de actuación. Son múltiples los beneficios que pueden obtenerse con la revalorización de la participación académica y empresarial.
Sin embargo, ¿existe en la actualidad una cultura constructiva de apoyo mutuo que pueda crecer hasta consolidarse totalmente en los próximos años?, ¿los académicos propiciamos escenarios de participación pertinentes con una visión hacia el futuro?, ¿diseñamos planes de vinculación o intervenimos como producto de la casualidad?, ¿aprovechamos la tecnología y nuestras fortalezas académicas para ayudar a empresas y emprendedores a adquirir ventajas competitivas? Estos son algunos aspectos que merecen reflexionarse para conocer más sobre nuestra realidad actual y prepararnos para los retos que ya hemos comenzado a enfrentar.

\section{Referencias}

Alonso-Almeida, M. d. M. y Llach, J. (2013). Adoption and use of technology in small business environments. The service industries journal, 33(15-16), 1456-1472.

Dada, O. y Fogg, H. (2016). Organizational learning entrepreneurial orientation, and the role of university engagement in smes. International Small Business Journal, 34(1), 86-104.

Deloitte (2010). What Cloud Computing means for business, and how to capitalize on it Deloitte.

Ding, Z., Li Sun, S. y Au, K. (2014). Angel inverstor's selection criteria: A comparative institutional perspective. Asia Pacific Journal of Management, 31, 705-731.

Karimi, S., Biemans, H. J. A., Lans, T. y Mohamnad, C., Mulder, M. (2016). The impact of entrepreneurship education: A study of iranian students' entrepreneurial intentions and opportunity identification.

Journal of Small Business Management, 54(1), 187-209.

Kendall, E. y Kendall, J. E. (2013). Systems Analysis and Design. United States: Prentice Hall.

Martínez-Caro, E. y Cegarra-Navarro, J. G. (2012). El desarrollo de competencias transversales mediante proyectos de emprendimiento en el marco de una asignatura de dirección de operaciones. Working Papers on Operations Management, 3(2), 9-31.

Messerschmitt, D. y Stuck, B. (2008). Effective communication: The what, why, and how of entrepreneurship. IEEE Signal Processing Magazine, 25(4), 105-109.

Premand, P., Brodman, S., Almeida, R., Grun, R. y Barouni, M. (2016). Entrepreneurship education and entry into self- employment among university graduates. World Development, 77, 311-327.

Rhoades, G. y Slaughter, S. (1991). Administrators and patents: The negotiation of technology transfer. Sociology of Education, 64(2), 65-77.

Ruiz Jiménez, J. M., Cabeza Pulles, D. y Briano Turrent, G. d. C. (2012). Un caso de estudio en la Facultad de Ciencias Económicas y Empresariales de la UGR. ReiDoCrea. Revista electrónica de investigación Docencia Creativa, 1, 144-157.

Urueña, A., Ferrarie, A., Blanco, D. y Valdecasa, E. (2012). Cloud Computing: retos y oportunidades.España.

Wang, S. y Wang, H. (2015). Design and delivery of a new course of information technology for small business. Journal of Information Systems Education, 26(1), 37-46. 